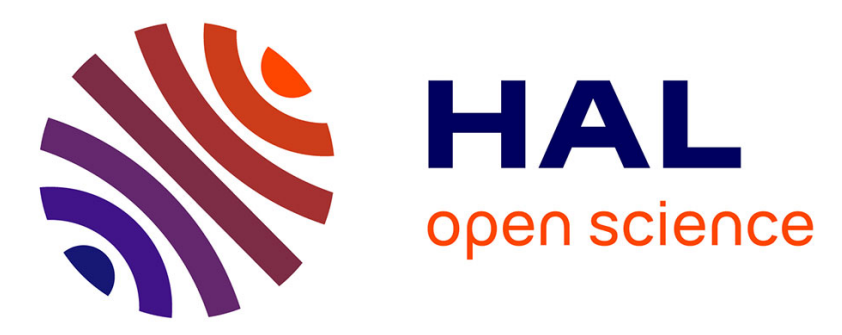

\title{
Proposition d'une typologie d'acheteurs alimentaires selon leur rapport au temps et à la technologie
}

\author{
Andréa Gourmelen, Jeanne Lallement
}

\section{To cite this version:}

Andréa Gourmelen, Jeanne Lallement. Proposition d'une typologie d'acheteurs alimentaires selon leur rapport au temps et à la technologie. Revue management \& avenir, 2016, 89, pp.129-151. 10.3917/mav.089.0129 . hal-01488473

\section{HAL Id: hal-01488473 https://hal.science/hal-01488473}

Submitted on 4 Jan 2018

HAL is a multi-disciplinary open access archive for the deposit and dissemination of scientific research documents, whether they are published or not. The documents may come from teaching and research institutions in France or abroad, or from public or private research centers.
L'archive ouverte pluridisciplinaire HAL, est destinée au dépôt et à la diffusion de documents scientifiques de niveau recherche, publiés ou non, émanant des établissements d'enseignement et de recherche français ou étrangers, des laboratoires publics ou privés. 


\title{
Proposition d'une typologie d'acheteurs alimentaires selon leur rapport au temps et à la technologie ${ }^{1}$
}

\author{
Andréa Gourmelen \\ Maître de conférences \\ Université Montpellier, IUT Montpellier-Sète, Laboratoire MRM (EA4557) \\ andrea.gourmelen@umontpellier.fr
}

Jeanne Lallement

Maître de conférences

Université La Rochelle, IUT La Rochelle, Laboratoire CEREGE LR Mos (EA1722)

jeanne.lallement@univ-lr.fr

\section{Résumé :}

A l'heure où la grande distribution crée des services technologiques permettant au consommateur de "gagner du temps" (drive, self-scanning...), cette recherche propose une typologie des acheteurs alimentaires fondée sur leur temps subjectif (la pression temporelle chronique - PTC) et leur rapport à la technologie. Une enquête portant sur 289 répondants dresse 5 profils de consommateurs : les familles tiraillées, les speed technophobes, les jeunes adeptes du dépannage, les zens traditionnels et les geeks, permettant aux distributeurs un ciblage précis et opérationnel.

Mots-clés : Pression temporelle, technologie, typologie, courses alimentaires

\section{A typology of grocery shoppers based on their attitudes toward time and technology}

\begin{abstract}
:
As major retailers launch technological solutions to «save time » for their consumers (drive, self-scanning...) to save time, this research develops a consumer typology founded on both subjective time (chronic time pressure) and attitudes towards technology. A survey based on 289 respondents allows us to sets up 5 classes of consumers: "torn families", "hurry technophobe", "young last-minute shoppers", "cool traditional" and "geek". This typology results in a more accurate targeting of consumers by the retailers.

Key-words: time pressure, technology usage, typology, grocery shopping
\end{abstract}

\footnotetext{
${ }^{1}$ Cet article est issu d'une communication retravaillée suite au congrès de l'association française du marketing (AFM 2015)
} 


\section{Introduction}

"Depuis que Lucie a découvert Carrefour drive, elle passe beaucoup moins de temps à faire ses courses $»{ }^{2}$ Néanmoins, pour gagner du temps, Lucie a aujourd'hui l'embarras du choix entre diverses formules de magasinage. Le temps des consommateurs est une préoccupation actuelle des distributeurs alimentaires. Ces derniers ont multiplié les services proposant un « gain de temps ». On trouve cette idée dans le mode d'accès au consommateur, qui peut être plus rapide, comme dans le drive et les formats de proximité aux noms parfois évocateurs («U Express », «Intermarché Express »), ainsi que dans les technologies de vente, annoncées comme plus rapides (self-scanning et caisses express). Pour cause, l'impression de manque de temps au quotidien et la volonté d'en gagner à tout prix sont des phénomènes vécus par de nombreux individus. Avec la diffusion massive des technologies de l'information, la pression quotidienne est de plus en plus intense sur tout ce « qui va plus vite » (Leccardi, 2011), soulignant une impression permanente de temps qui fait défaut (Rosa, 2010). La distribution propose plusieurs réponses à ce phénomène. Les hypermarchés permettent une nouvelle approche temporelle des courses : sans attendre aux caisses (la caisse express), sans passer par les caisses (le self-scanning), ou sans passer par les rayons (le drive). Ces self-services technologies peuvent être perçues comme ludiques et attractives (Mencarelli et Rivière, 2014), ce qui permet de diminuer le côté «corvée » que représente les courses alimentaires pour certains consommateurs. En outre, la technologie perçue en magasin a un impact sur le temps passé perçu en magasin (Goudey, 2013). Paradoxalement, les hypermarchés, conçus historiquement dans une optique de gain de temps («tout sous le même toit ») par rapport aux magasins de proximité, se voient aujourd'hui concurrencés par ces derniers à ce propos. Certes, moins de self-services technologies y sont présentes, mais la simplification des choix induite par les magasins de proximité et leur localisation peuvent répondre au besoin de gain de temps d'un consommateur toujours plus pressé (Gahinet, 2014).

En ce sens, les distributeurs donnent l'impression d'une segmentation binaire des consommateurs. Il y aurait d'un côté les consommateurs pressés en permanence, cœur de cible de la grande distribution, qui leur adresse toujours plus d'offres censées répondre à un besoin de «gain de temps» (Renko et Druzijanic, 2014) et de l'autre les consommateurs "non pressés », se contentant des caisses traditionnelles, voire des marchés. Cette segmentation binaire (pressés/non pressés) semble un peu simplificatrice de la réalité. La littérature a d'ores et déjà montré la diversité des acheteurs par le biais de typologies (Reynolds et al, 2002 ; Ruiz et al, 2004 ; Bosnjak, Galesic et Tuten, 2007). Cependant, elles n'expliqueraient qu'en partie le choix d'un mode de magasinage plutôt qu'un autre en ne tenant pas compte du temps subjectif des consommateurs. Cela peut sembler paradoxal au regard des arguments temporels des distributeurs alimentaires. Faut-il nécessairement être pressé pour adopter les innovations de la distribution ? L'attitude à l'égard de la technologie joue-t-elle un rôle dans le choix d'un mode de magasinage alimentaire en complément du fait d'être pressé ou non ? Cette recherche tente d'apporter des réponses à ces interrogations en dressant une typologie d'acheteurs alimentaires, sur la base de deux variables psychologiques (traits de personnalité stables) : la pression temporelle chronique et l'attitude à l'égard de la technologie. La première reflète le sentiment permanent d'un temps insuffisant; la seconde le degré d'acceptation des technologies dans la vie quotidienne. A la suite d'une revue de la littérature montrant l'intérêt de mobiliser des traits de personnalité stables en rapport avec le temps subjectif et le rapport à la technologie pour expliquer les comportements de magasinage, une typologie d'acheteurs alimentaires est ainsi proposée.

\footnotetext{
${ }^{2}$ Extrait d'une vidéo Youtube de Carrefour Drive : https://www.youtube.com/watch?v=Tn9qgIoEE2A
} 


\section{Le choix d'un mode de magasinage : une approche par des traits de personnalité et dispositions stables}

Dans la littérature relative au magasinage, la plupart des travaux s'intéressant au temps ou à la technologie, mobilisent des variables reflétant des états éphémères dans lesquels se trouvent ponctuellement les consommateurs (ex : consommateur pressé pour effectuer un achat particulier, opinions relatives à une technologie présente en magasin). A contrario, les traits de personnalité stables sont peu mobilisés. La pression temporelle chronique et l'attitude à l'égard de la technologie reflètent respectivement une vision subjective stable de l'écoulement du temps au quotidien et une appétence générale pour les technologies. Ces traits peuvent influencer de manière permanente les comportements de magasinage alimentaire.

\subsection{La pression temporelle chronique (PTC), un manque de temps permanent}

Le temps des consommateurs est étudié de manière pluridisciplinaire (économie, sociologie, psychologie, sciences de gestion) depuis plus de 40 ans. En marketing, à partir des travaux nord-américains de Wright (1974), de Jacoby et al (1976) et de Berry (1979), le temps est devenu un objet d'étude à part entière. Plus spécifiquement, les recherches en distribution et marketing des services s'intéressent au temps objectif, c'est-à-dire la quantité de temps disponible du consommateur (Gautschi, 1981; McDonald, 1994 ; Herrington et Capella, 1995 ; Gallouj, 2006) et aux variables situationnelles liées au temps subjectif. Il s'agit notamment de la perception d'une durée passée dans un point de vente ou du fait d'être ponctuellement pressé à un moment donné. Ainsi, l'atmosphère du point de vente (Daucé et Rieunier, 2002) et le degré de technologie perçue en magasin (Goudey, 2013) influencent la perception du temps passé en magasin. Avec l'essor du commerce électronique, de plus en plus d'auteurs ont cherché à faire le lien entre le temps subjectif du consommateur et son comportement de magasinage. Internet, en brisant les frontières spatiales et temporelles, modifie la façon dont les différentes tâches sont planifiées et la façon dont le temps est perçu (Lee et Whitley, 2002). Quant au temps subjectif lui-même, il influence à son tour le comportement de magasinage. En fonction de sa perception individuelle du temps quotidien disponible, le consommateur ne fera pas les courses de la même façon. A ce propos, la pression temporelle semble une notion importante dans le choix du mode de magasinage.

La littérature marketing distingue trois types de pression temporelle : situationnelle, chronique et ultime (Lallement, 2010 ; Gourmelen, Urien et Le Gall-Ely, 2016). La pression temporelle situationnelle (fait d'être ponctuellement pressé en raison d'un temps limité pour effectuer un achat), la plus mobilisée dans la littérature en distribution, est reconnue comme une variable modératrice du comportement en magasin. Les recherches antérieures montrent que les clients pressés font moins d'achats impulsifs (Iyer, 1989 ; Beatty et Ferrell, 1998), oublient plus facilement les achats prévus (Park, Iyer et Smith, 1989) et sont plus sensibles aux prix (Lallement et Zollinger, 2013). Cependant, cette pression temporelle étudiée ne représente qu'un état éphémère dans lequel se trouvent les consommateurs. Peu étudié dans la littérature en distribution, le temps subjectif concerne des traits psychologiques, internes à l'individu, relatant une préférence stable, telle que l'orientation «passé » «présent» ou «futur» (Bergadaà, 1988, 1989), reflétant une préférence pour une zone temporelle. Cette recherche s'intéresse particulièrement au consommateur pressé en permanence, cible des distributeurs alimentaires avec la pression temporelle chronique (PTC). Celle-ci se définit comme une « lutte chronique, incessante pour achever de plus en plus de choses en moins en moins de temps » (Rizkalla, 1989). Elle est ressentie de façon continuelle dans la vie quotidienne et se compose d'un volet cognitif (la conscience d'un manque de temps permanent) et d'un volet 
affectif (les sentiments qui l'accompagnent) (Szollos, 2009 ; Lallement et Machat, 2011). Deux sentiments opposés (positifs et négatifs) sont associés à la PTC. Les sentiments sont négatifs (panique, tristesse) lorsque l'individu a l'impression qu'il subit en permanence une contrainte temporelle. Pour d'autres individus, au contraire, la PTC est associée à des affects positifs. Aubert (2003) reconnait que la société moderne est «malade du temps », mais que cette expérience est pour les "shootés à l'urgence », une construction mentale, une forme de démonstration de leur propre supériorité et de leur puissance d'appropriation du temps. La PTC est alors un état choisi (Rastegary et Landy, 1993 ; Lallement et Machat, 2011). Au regard de sa définition, il semble intéressant de mobiliser cette variable en raison de sa stabilité (par rapport à la pression temporelle situationnelle). Pourtant, la pression temporelle chronique reste peu étudiée dans un contexte de magasinage. Kim et Kim (2008), prennent néanmoins en compte une pression temporelle permanente et indiquent que celle-ci modère la relation entre le plaisir au shopping et le comportement. Chetthamrongchai et Davies (2000) mobilisent également la PTC dans leur typologie d'acheteurs et obtiennent un profil de consommateurs pressés, à la recherche de commodités. Ils observent que ces derniers détestent les courses alimentaires. Or, les formules proposées par la distribution ont évolué depuis cette typologie. Elles intègrent des technologies au service de l'ergonomie (Goudey, 2013), telles que le self-scanning ou les caisses express. Nous étudions ici simultanément le fait d'être pressé en permanence (à travers la PTC) et le rapport qu'entretiennent les individus avec les nouvelles technologies.

\subsection{L'attitude à l'égard de la technologie, variable complémentaire au rapport au temps}

Les recherches traitant de la technologie en marketing s'intéressent principalement aux innovations mobilisant des technologies et à leur perception (Goudey, 2013). Ces innovations interviennent à diverses étapes du processus d'achat. Dans la grande distribution, les technologies peuvent concerner le mode d'accès au client (drive), ou la vente au sens strict du terme (self-scanning, caisses express). Le drive compte 3569 points de vente en France ${ }^{3}$. Pourtant, certains experts soulignent son ambiguïté : "Les gens sont satisfaits, ils sont rassurés par ce qu'ils y trouvent : le gain de temps, le service, etc... Malgré tout, au-delà de cette satisfaction globale, il y a une grosse déperdition à cause de la navigation pas claire. A un point où certains renoncent tout simplement à y faire leurs courses »(Jean Gomez, directeur marketing de Yuseo $)^{4}$. Le consommateur gagne t-il réellement du temps s'il se perd sur le site web ? Parallèlement, les enseignes proposent aux consommateurs de gagner du temps en utilisant une formule moins technologique : le magasin de proximité. La taille du magasin et son assortiment sont mis en avant comme facteurs de gain de temps. Le gain de temps apparaît également comme un argument pour inciter les consommateurs à utiliser les technologies disponibles au sein du point de vente. La France compte plus de 10.000 caisses express, auxquelles il faut rajouter les systèmes de scannette pour enregistrer soi-même ses courses. Ces innovations reposent sur une coproduction de la part du client, qui prend une part importante dans le processus de délivrance du service. L'argument essentiel de la grande distribution dans l'usage des self-services technologies réside dans la diminution du temps d'attente (Meuter et al, 2000 ; Dabholkar, Bobbitt et Lee, 2003). Pourtant, ces avantages sur la perception d'une réduction perçue du temps d'attente ne sont pas validés dans la littérature (Weijters et al, 2007). Considérer uniquement le temps subjectif semble alors insuffisant.

\footnotetext{
${ }^{3} \mathrm{http}$ ://www.lsa-conso.fr/drive-603-nouvelles-unites-ont-ete-creees-en-2014-selon-lsa-expert,198901

${ }^{4}$ http://www.lsa-conso.fr/drive-10-cles-pour-comprendre-l-experience-client-infographie,199600
} 
La difficulté de prouver le gain de temps induit par les technologies s'éclaire à l'aube des travaux académiques s'intéressant aux antécédents de l'adoption des innovations de la grande distribution. Certaines recherches se sont intéressées aux résistances d'un consommateur adoptant des attitudes de rejet ou d'évitement de ces innovations, conscient des risques potentiels sur l'emploi (Coutelle et Desgarets, 2013). D'autres soulignent les différences générationnelles, d'où le nécessaire accompagnement des enseignes pour assurer la montée en compétences des consommateurs (Collin-Lachaud et Longo, 2014). Ainsi, la maturité technologique du consommateur jouerait un rôle dans la qualité perçue du self-service et son intention de l'utiliser (Lin et Hsieh, 2006). A l'inverse, les consommateurs à l'attitude négative envers la technologie en général (Rosen et al, 2013), pourraient être moins enclins à utiliser ces innovations. Dans une étude exploratoire, Mencarelli et Rivière (2014) montrent que les self-services technologies peuvent être perçues comme engendrant un gain ou une perte de temps selon les utilisateurs. Si ces auteurs montrent l'existence de différences interindividuelles en matière de valeur perçue des self-services technologies, ils ne mobilisent que des variables relatives à la perception de ces innovations pour les expliquer.

Comme pour le temps subjectif, ce qui est étudié dans ces recherches est un état éphémère. Il s'agit de la perception d'une technologie développée dans un point de vente particulier; au détriment de l'attitude générale des consommateurs à l'égard des technologies. Cependant, en France, si la technologie est ancrée dans la vie quotidienne, elle n'est pas forcément acceptée par tous, mais appréciée à des degrés divers. En ce sens, Rosen et al (2013) évoquent l'intérêt de mobiliser l'attitude à l'égard de la technologie plutôt qu'un nombre d'heures passé à utiliser les technologies. En effet, certains individus ont l'impression qu'avec la technologie, tout est possible et donc que cette dernière est importante. Inversement, elle peut être perçue négativement (ex : comme facteur d'isolement), ou entraîner une dépendance.

En synthèse de cette revue de littérature, il semble intéressant :

- De mobiliser l'attitude générale à l'égard de la technologie, disposition stable, pour comprendre l'utilisation ou non des innovations technologiques de la distribution.

D'étudier conjointement le rapport au temps et à la technologie car l'argument principal des distributeurs est le gain de temps pour le consommateur, indépendamment du mode de magasinage promu. Ce gain de temps serait possible grâce à des formules de distribution plus ou moins technologiques à divers stades du processus d'achat.

Comment les consommateurs, selon leur PTC plus ou moins forte, subie ou choisie, s'approprient les innovations technologiques censées leur faire gagner du temps ? Existe-t-il des consommateurs pressés, mais technophobes, refusant d'utiliser ces innovations technologiques? La typologie proposée dans la partie suivante approfondit les liens entre le mode de magasinage, le rapport à la technologie et les dimensions de la PTC, et permet ainsi d'enrichir la compréhension de l'adoption des innovations de la distribution.

\section{Méthodologie de la recherche}

Suite à la revue de la littérature, qui souligne l'intérêt de mobiliser des variables psychologiques conjointement au comportement de magasinage, nous avons opté pour une segmentation a posteriori. L'idée est, à partir de ces variables, de définir des groupes de consommateurs semblables qu'il serait intéressant de mieux comprendre pour les distributeurs, dans une optique de ciblage. 


\subsection{Collecte des données}

La collecte de données a été réalisée par le biais d'un questionnaire en ligne. L'échantillon, constitué par effet boule de neige, est composé de 289 personnes âgées de 18 à 78 ans (âge moyen $=32,5$ ans ; âge médian $=29$ ans) et effectuant des courses alimentaires. Nous avons veillé à une répartition équitable entre générations pour tenir compte de la familiarité avec les nouvelles technologies $(34,4 \%$ de génération $\mathrm{X}$ ou antérieure, $35,4 \%$ de $\mathrm{Y}$ et $30,2 \%$ de $\mathrm{Z}$, les «Digital Native $»^{5}$ ). Concernant le mode de magasinage, notre échantillon est constitué de $62 \%$ d'individus n'ayant jamais utilisé le drive, ce qui est cohérent avec l'étude de Fauconnier (2014) dans l'Observatoire Neopost ID du Click and collect. Ainsi, l'échantillon est représentatif de la population effectuant des courses alimentaires au regard de ces critères.

\subsection{Validation des instruments de mesure}

Nous avons choisi de tenir compte des différentes habitudes en termes de modes de magasinage en proposant 6 modes de distribution alimentaires plus ou moins technologiques dans le mode d'accès au client (le drive, les magasins de proximité et les marchés ou ventes directes) ou dans la vente au sens strict du terme (le self-scanning, les caisses express, les caisses traditionnelles). Les répondants étaient amenés à s'exprimer sur leur fréquence et la situation d'utilisation de chacun de ces modes via une échelle de Likert en 5 points. Le questionnaire comprenait également des variables socio-démographiques (âge, genre, niveau d'études, nombre de personnes dans le foyer, CSP). Concernant les variables psychographiques, nous avons utilisé deux échelles issues de la littérature existante :

- Pression temporelle chronique (Gourmelen, 2013). Cette échelle combine les échelles de Lallement et Machat (2011) et de Kaufman-Scarborough et Lindquist (2003), ce qui permet de refléter l'aspect bidimensionnel (cognitif et affectif) de la PTC.

- Attitude à l'égard de la technologie (Rosen et al, 2013). Compte-tenu des innovations de la distribution étudiées, nous avons fait rétrotraduire cette échelle constituée de trois dimensions : attitude positive, négative et anxiété/dépendance vis-à-vis de la technologie.

Après vérification de la normalité des données (Skewness $<|1.5|$ et Kurtosis $<|1|$ ), des analyses en composantes principales (ACP) ont été effectuées à l'aide du logiciel SPSS $22^{6}$ (tableau 1). L'échelle de PTC obtenue se caractérise par une structure bidimensionnelle (dimension cognitive : le manque de temps permanent et dimension affective : la PTC choisie) sans épuration nécessaire. En revanche, une structure bidimensionnelle (et non tridimensionnelle) pour l'attitude à l'égard de la technologie est constatée. La dimension nommée «dépendance assumée » allie les items les plus discriminants (les moins généraux) de l'attitude positive avec ceux décrivant une « anxiété/dépendance ». La dimension « attitude négative » est identique à celle obtenue par Rosen et al (2013).

\footnotetext{
${ }^{5}$ Notons que les bornes délimitant les générations $\mathrm{Y}$ et $\mathrm{Z}$ demeurent à l'heure actuelle relativement floues en termes d'années de naissance (1980 à 2002 selon les auteurs). Etant donné que le taux d'équipement en ordinateurs fixes et l'accès internet s'est accéléré au milieu des années 90 en France, nous avons choisi de considérer comme «digital native » les personnes nées après 1990.

${ }^{6}$ Sélection des facteurs dont les valeurs propres sont supérieures à 1 et élimination des items ne s'agrégeant sur aucun axe ou au contraire sur plusieurs facteurs de manière comparable.
} 


\begin{tabular}{|c|c|c|c|c|c|}
\hline Construit & Item & $\begin{array}{c}\text { Poids } \\
\text { factoriel }\end{array}$ & $\begin{array}{l}\text { Valeur } \\
\text { propre }\end{array}$ & $\begin{array}{c}\text { variance } \\
\text { expliquée }\end{array}$ & $\alpha$ \\
\hline \multirow{6}{*}{$\begin{array}{c}\text { PTC - } \\
\text { Dimension } \\
\text { cognitive }\end{array}$} & $\begin{array}{l}\text { J'ai parfois le sentiment qu'il n'y a pas assez d'heures } \\
\text { dans la journée }\end{array}$ & 0,84 & \multirow{6}{*}{3,25} & \multirow{6}{*}{$30 \%$} & \multirow{6}{*}{0,8} \\
\hline & $\begin{array}{l}\text { Il me faudrait plus d'heures dans la journée pour faire } \\
\text { tout ce qu'on attend de moi }\end{array}$ & 0,79 & & & \\
\hline & Quand vous sentez-vous pressé(e) par le temps $?^{7}$ & 0,77 & & & \\
\hline & J'ai l'impression de ne jamais avoir de temps pour moi & 0,76 & & & \\
\hline & $\begin{array}{l}\text { Je suis trop sollicité(e) par rapport au temps dont je } \\
\text { dispose }\end{array}$ & 0,75 & & & \\
\hline & J'ai l'impression de ne jamais parvenir à tout faire & 0,66 & & & \\
\hline \multirow{5}{*}{$\begin{array}{c}\text { PTC - } \\
\text { Dimension } \\
\text { affective }\end{array}$} & Cela me plaît d'être pressé(e) & 0,93 & \multirow{5}{*}{3,95} & \multirow{5}{*}{$35,9 \%$} & \multirow{5}{*}{0,91} \\
\hline & Je préfère être pressé(e) & 0,88 & & & \\
\hline & J'aime me sentir pressé(e) dans ma vie quotidienne & 0,86 & & & \\
\hline & Etre pressé(e), c'est stimulant & 0,80 & & & \\
\hline & C'est mon choix que d'être pressé(e) & 0,78 & & & \\
\hline \multirow{5}{*}{$\begin{array}{c}\text { Dépendance } \\
\text { assumée à } \\
\text { la } \\
\text { technologie }\end{array}$} & $\begin{array}{l}\text { Je suis anxieux quand je n'ai pas Internet à } \\
\text { disposition }\end{array}$ & 0,83 & \multirow{5}{*}{3,53} & \multirow{5}{*}{$37,5 \%$} & \multirow{5}{*}{0,84} \\
\hline & $\begin{array}{l}\text { Je suis anxieux quand je n'ai pas mon téléphone } \\
\text { portable }\end{array}$ & 0,79 & & & \\
\hline & Je suis dépendant de la technologie & 0,79 & & & \\
\hline & $\begin{array}{l}\text { Je trouve qu'il est important d'avoir internet à } \\
\text { n'importe quel moment }\end{array}$ & 0,78 & & & \\
\hline & $\begin{array}{l}\text { Je pense qu'il est important de se tenir au courant des } \\
\text { dernières tendances en matière de technologie }\end{array}$ & 0,61 & & & \\
\hline \multirow{3}{*}{$\begin{array}{c}\text { Attitude } \\
\text { négative à } \\
\text { l'égard de } \\
\text { la } \\
\text { technologie }\end{array}$} & $\begin{array}{l}\text { Les nouvelles technologies rendent la vie plus } \\
\text { compliquée }\end{array}$ & 0,82 & \multirow{3}{*}{1,41} & \multirow{3}{*}{$24,3 \%$} & \multirow{3}{*}{0,68} \\
\hline & $\begin{array}{l}\text { Les nouvelles technologies font perdre trop de temps } \\
\text { aux gens }\end{array}$ & 0,79 & & & \\
\hline & Les nouvelles technologies isolent les gens & 0,68 & & & \\
\hline
\end{tabular}

\section{Tableau 1 - Analyses en composantes principales}

\subsection{Analyses typologiques}

L'analyse typologique permet de regrouper les individus en fonction de leurs points communs. Pour ce faire, le chercheur est libre de choisir les critères déterminant l'appartenance à un groupe. Dans notre contexte, les services proposés par les enseignes de distribution sont censés faire gagner du temps au consommateur et intègrent des éléments technologiques. Ainsi, la typologie s'est effectuée sur la base des fréquences d'utilisation des modes de magasinage identifiés et des scores factoriels issus des ACP réalisées sur les échelles évoquées précédemment. Pour éviter la corrélation entre dimensions d'une même échelle, nous avons opté pour des rotations Varimax. Nous avons ensuite procédé en deux étapes (Hair et al, 2010, p537). La première étape a consisté en une classification hiérarchique ascendante pour déterminer un nombre de groupes (méthode de Ward). Pour ce faire, nous avons scindé notre échantillon en 3 groupes aléatoires ${ }^{8}$. Les dendrogrammes montrent une certaine stabilité des structures en 5 et 6 groupes (présence dans la majorité des échantillons et absence de variation brutale de l'indice de fusion). Dans la seconde étape (validation/interprétation des groupes), nous avons utilisé une classification non-hiérarchique (K-means) en fixant le nombre de groupes à 6 puis à 5 . La classification en 5 groupes est apparue la plus adaptée car la taille de chaque groupe est supérieure à $10 \%$ de l'effectif total, contrairement à la

\footnotetext{
${ }^{7}$ Les modalités de réponse sont ici proposées sous forme d'une échelle de 1 à 5 , de 1 « jamais » à 5 « toujours »

${ }^{8}$ E1, E2, E3, respectivement constitués de 96, 96 et 97 individus.
} 
classification en 6 groupes. Afin d'interpréter au mieux la typologie, nous avons ajouté des variables «muettes » sociodémographiques (âge, sexe, génération, niveau d'études...) et d'autres liées aux modes de magasinage (mode le plus utilisé en fonction de diverses situations d'achat). Ces situations ont été inspirées des «projets d'usage du cybermarché et autres formats » observés par Picot-Coupey, Cliquet et Petr (2010). Le test bilatéral d'égalité a permis d'appréhender les écarts entre les groupes.

\section{Résultats : une typologie d'acheteurs alimentaires en 5 profils}

L'analyse typologique donne différents profils riches d'enseignements liant formule de distribution et perception du temps (tableau 2). Dans un but de compréhension, nous avons nommé chaque profil par un adjectif ou une expression le caractérisant.

\subsection{Les familles tiraillées (12\% de l'échantillon)}

Ce groupe, constitué essentiellement de CSP+ et de familles (nombre de personnes au foyer > 3 , le plus élevé des groupes), se démarque par sa très forte utilisation du drive, formule privilégiée de manière générale, mais aussi favorite pour le plein hebdomadaire et l'achat de produits lourds ou encombrants. Le drive reste néanmoins la seule formule de distribution technologique utilisée, la scannette et les caisses express les attirent peu, contrairement à d'autres formules plus classiques, telles que les marchés ou ventes directes et magasins de proximité. Ces familles pressées en permanence mais n'aimant pas cette façon de vivre, semblent ressentir un besoin de gagner du temps dans leurs courses alimentaires, comblé avec la rapidité des modes d'accès que sont le drive et les magasins de proximité, ce qui apparait cohérent avec la littérature sur le sujet (Gahinet, 2014). De plus, leur attitude à l'égard de la technologie complète ce constat. Dépendants à la technologie dans leur vie à cent à l'heure, ces individus développent cependant une attitude négative à son égard. Ils n'assument pas cette dépendance et évitent ainsi les modes de distribution trop technologiques, exception faite du drive pour la corvée du plein hebdomadaire en raison de sa praticité. Pour le reste, marchés et magasins de proximité trouvent grâce à leurs yeux, car permettant de débrancher de la vie quotidienne.

\subsection{Les speed technophobes ( $26 \%$ de l'échantillon)}

A l'opposition du groupe précédent, ce groupe, qui ne se démarque pas par des caractéristiques socio-démographiques particulières, comprend les plus faibles utilisateurs du drive et des magasins de proximité. "Speed» et fiers de l'être, ces consommateurs sont toujours pressés. La pression temporelle est alors vécue comme un choix de vie, assimilée à des affects positifs. Ce groupe apparaît relativement dépendant de la technologie, même si elle n'est pas appréciée. Ces consommateurs pressés vivent dans un monde technologique auquel ils ne s'identifient pas toujours. Les courses sont-elles alors un moyen de « déconnecter », une coupure avec la techno-dépendance ? En effet, la formule la plus adaptée pour faire les courses consiste pour eux à utiliser les caisses classiques ou, dans une moindre mesure, se rendre au marché. Dans leur situation, il y a peu de place pour les innovations de la grande distribution, exception faite pour les caisses express qui concurrencent les caisses classiques dans la situation d'achat de dépannage. Le reste du temps, ils choisissent d'être « débranchés » de ces innovations de la distribution, comme ils ont choisi d'être pressés dans leur quotidien. Tester des modes de distribution plus novateurs semble ainsi une perte de temps pour ces consommateurs pressés, qui se dirigent systématiquement vers les caisses classiques (même en cas de produits encombrants), qu'ils connaissent bien. 


\subsection{Les jeunes adeptes du dépannage ( $25 \%$ de l'échantillon)}

Ce groupe, à la plus faible moyenne d'âge, assume à la fois un goût pour les nouvelles technologies et une dépendance à celles-ci. Peu pressés et n'appréciant pas la pression temporelle, les individus composant ce groupe apparaissent néanmoins attirés par les innovations «express» de la distribution. En effet, ces consommateurs adoptent principalement la caisse express pour les courses de dépannage et les magasins de proximité. Paradoxalement, malgré un goût prononcé pour la technologie, ces individus délaissent le drive. Ce dernier implique notamment un panier important en valeur et/ou en volume, ainsi qu'une certaine organisation pour faire le «plein» hebdomadaire. Ces caractéristiques du drive ne semblent pas en phase avec le mode de vie de ces jeunes adultes, effectuant généralement leurs courses alimentaires au jour le jour (le dépannage semble la règle) et ne possédant pas forcément de véhicule. Ces choix de magasinage semblent ainsi cohérents avec la structure familiale et la période de vie observée dans ce groupe (prédominance de célibataires, groupe qui comporte significativement le plus d'étudiants).

\subsection{Les zens traditionnels ( $27 \%$ de l'échantillon)}

Ce groupe, à la moyenne d'âge la plus élevée, est composé d'individus ni pressés, ni technodépendants. De manière logique, ils refusent toutes les innovations technologiques de la distribution censées faire gagner du temps et ce, quelle que soit la situation d'achat (caisses traditionnelles privilégiées pour le plein, les produits encombrants et les courses de dépannage). Ainsi, ces individus ne se sentent pas pressés, ils aiment prendre leur temps. Pour eux, les courses alimentaires riment avec les magasins de proximité, les caisses traditionnelles et les marchés ; tout ce qui n'implique pas de technologie. En effet, la technologie est peu entrée dans leur quotidien, ils ne ressentent aucune dépendance vis-à-vis d'elle. Il en découle une absence d'intérêt pour les nouveaux modes de magasinage (drive, caisses express, selfscanning), ne correspondant pas à leur mode de vie ; et ce, d'autant plus qu'ils ne sont pas concernés par l'argument de gain de temps mis en avant par les distributeurs. En effet, ils ne sont pas pressés par le temps donc ne sont pas nécessairement intéressés pour en gagner.

\subsection{Les geeks (10\% de l'échantillon)}

Les individus composant ce groupe apprécient la technologie sans pour autant se sentir dépendants d'elle et ne ressentent pas particulièrement la pression temporelle comme une caractéristique de leur vie. Les modes de magasinage adoptés leur permettent-ils de réduire la pression du temps par une meilleure gestion de celui-ci ? En effet, ce sont ceux qui utilisent le plus les innovations technologiques des distributeurs. Très adeptes du self-scanning, des caisses express et du drive, ces «aventuriers » des innovations semblent tout tester et tout adopter. Si ce comportement d'exploration n'est pas antinomique avec des modes de magasinage traditionnels, ce groupe se démarque de par sa fréquence d'utilisation des caisses classiques, significativement la plus faible. Dans leur comportement multiple, lié aux circonstances de consommation, le drive reste leur mode préféré, en particulier dans le cas de produits encombrants et pour le plein hebdomadaire. Les caisses express sont privilégiées lors d'achats de dépannage. Ces choix semblent ainsi davantage dictés par la facilité et la praticité que par la recherche de gain de temps. 


\begin{tabular}{|c|c|c|c|c|c|c|c|c|c|c|c|}
\hline & & \multicolumn{10}{|c|}{ Typologie d'acheteurs alimentaires ${ }^{9}$} \\
\hline & & \multicolumn{2}{|c|}{ 1- Familles tiraillées } & \multicolumn{2}{|c|}{ 2-Speed technophobes } & \multicolumn{2}{|c|}{ 3-Jeunes adeptes du dépannage } & \multicolumn{2}{|c|}{ 4-Zens traditionnels } & \multicolumn{2}{|c|}{5 - Geeks } \\
\hline & & \begin{tabular}{|l|} 
Moyenne \\
\end{tabular} & Nombre & Moyenne & Nombre & Moyenne & Nombre & Moyenne & Nombre & Moyenne & Nombre \\
\hline \multicolumn{12}{|c|}{ Variables actives de la typologie } \\
\hline \multicolumn{2}{|c|}{$\begin{array}{c}\text { Relatives au comportement de } \\
\text { magasinage }\end{array}$} & & & & & & & & & & \\
\hline \multicolumn{2}{|c|}{ Fréquence drive } & $4,03 \mathrm{a}$ & & $1,24 b$ & & $1,31_{b}$ & & $1,28_{b}$ & & $3,34_{c}$ & \\
\hline \multicolumn{2}{|c|}{ Fréquence scannette } & $1,23_{\mathrm{a}, \mathrm{b}}$ & & $1,21_{a, b}$ & & $1,52_{\mathrm{a}}$ & & $1,12_{b}$ & & $3,00_{c}$ & \\
\hline \multicolumn{2}{|c|}{ Fréquence caisses express } & $2,66_{\mathrm{a}, \mathrm{d}}$ & & $2,62_{\mathrm{a}}$ & & $4,10_{b}$ & & $1,88_{c}$ & & $3,24_{d}$ & \\
\hline \multicolumn{2}{|c|}{ Fréquence caisses classiques } & $3,94 \mathrm{a}$ & & $4,61_{b}$ & & $4,20_{\mathrm{a}, \mathrm{c}}$ & & $4,54_{\mathrm{b}, \mathrm{c}}$ & & $2,83 d$ & \\
\hline \multicolumn{2}{|c|}{ Fréquence magasins de proximité } & $3,60_{a, c}$ & & $2,58 \mathrm{~b}$ & & $3,49 \mathrm{a}$ & & $4,08 \mathrm{c}$ & & $2,00_{b}$ & \\
\hline \multicolumn{2}{|c|}{ Fréquence marchés - ventes directes } & $3,666_{a}$ & & $2,92_{b}$ & & $1,72_{c}$ & & $3,00_{\mathrm{b}}$ & & $2,41_{b, c}$ & \\
\hline \multicolumn{12}{|c|}{ Relatives au rapport au temps quotidien } \\
\hline \multicolumn{2}{|c|}{ PTC-dim affective } & $-0,05_{\mathrm{a}, \mathrm{c}}$ & & $0,57_{b}$ & & $-0,21_{\mathrm{a}, \mathrm{c}}$ & & $-0,45 a$ & & $0,32_{b, c}$ & \\
\hline \multicolumn{2}{|c|}{ PTC-dim cognitive } & $0,29 \mathrm{a}, \mathrm{b}$ & & $0,39 \mathrm{a}$ & & $-0,10_{b, c}$ & & $-0,29 c$ & & $-0,36 b, c, d$ & \\
\hline \multicolumn{12}{|c|}{ Relatives au rapport à la technologie } \\
\hline \multicolumn{2}{|c|}{ Attitude/tech - Dépendance assumée } & $0,30_{\mathrm{a}, \mathrm{c}}$ & & $0,36_{a}$ & & $0,31_{\mathrm{a}, \mathrm{c}}$ & & $-0,69 b$ & & $-0,20_{b, c}$ & \\
\hline \multicolumn{2}{|c|}{ Attitude/tech - Attitude négative } & $0,36_{\mathrm{a}}$ & & $0,11_{\mathrm{a}}$ & & $-\mathbf{- 0 , 3 7 b}$ & & $0,26_{a}$ & & $-0,50_{b}$ & \\
\hline \multicolumn{12}{|c|}{ Variables « muettes » } \\
\hline \multicolumn{12}{|c|}{ Caractéristiques socio-démographiques } \\
\hline \multicolumn{2}{|l|}{ Age } & $35,00_{\mathrm{a}, \mathrm{d}, \mathrm{e}}$ & & $32,50_{a, b}$ & & $25,25 \mathrm{c}$ & & $38,77_{d}$ & & $29,90_{b, c, e}$ & \\
\hline \multicolumn{2}{|c|}{ Nombre de personnes / foyer } & $3,23 a$ & & $2,43_{\mathrm{a}, \mathrm{b}}$ & & $2,10_{b}$ & & $2,60_{a, b}$ & & $2,54_{a, b}$ & \\
\hline \multirow[t]{5}{*}{ Statut marital } & Célibataire & & $7 \mathrm{a}$ & & $32 \mathrm{a}$ & & $50_{b}$ & & $30_{\mathrm{a}}$ & & $10_{a}$ \\
\hline & Couple & & $11_{\mathrm{a}}$ & & $17 \mathrm{a}$ & & $13_{\mathrm{a}}$ & & $12_{\mathrm{a}}$ & & $9 \mathrm{a}$ \\
\hline & Marié & & $15_{\mathrm{a}}$ & & $21_{\mathrm{a}, \mathrm{b}}$ & & $7 \mathbf{b}$ & & $32_{\mathrm{a}}$ & & $10_{\mathrm{a}}$ \\
\hline & Divorcé & & $1_{\mathrm{a}}$ & & $2 \mathrm{a}$ & & $1_{\mathrm{a}}$ & & $2 \mathrm{a}$ & & 0 \\
\hline & Veuf & & 0 & & 0 & & 0 & & $1_{\mathrm{a}}$ & & 0 \\
\hline \multicolumn{2}{|c|}{ Variables « muettes » (suite) } & \multicolumn{2}{|c|}{1 - Familles tiraillées } & \multicolumn{2}{|c|}{ 2-Speed technophobes } & \multicolumn{2}{|c|}{3 - Jeunes adeptes du dépannage } & \multicolumn{2}{|c|}{ 4-Zens traditionnels } & $5-\mathbf{G}$ & \\
\hline
\end{tabular}

${ }^{9}$ Les valeurs dans la même ligne et sous-table ne partageant pas le même indice (a,b,c,d,e) diffèrent significativement à p<,05 dans le test bilatéral d'égalité pour proportions de colonne. Les cellules sans indice ne sont pas incluses dans le test. Les tests supposent des variances égales. Les tests sont ajustés pour toutes les comparaisons par paire au sein d'une ligne de chaque soustable le plus interne, à l'aide de la correction de Bonferroni. Lorsque les proportions de colonne sont égales à zéro, la comparaison n'a pas lieu. 


\begin{tabular}{|c|c|c|c|c|c|c|c|c|c|c|c|}
\hline \multicolumn{2}{|c|}{$\begin{array}{c}\text { Caractéristiques socio-démographiques } \\
\text { (suite) }\end{array}$} & \multirow[t]{2}{*}{ Moyenne } & \multirow{2}{*}{$\begin{array}{r}\text { Nombre } \\
0,00\end{array}$} & & \multirow{2}{*}{$\begin{array}{r}\text { Nombre } \\
0,00\end{array}$} & \multirow[t]{2}{*}{ Moyenne } & \multirow{2}{*}{$\begin{array}{r}\text { Nombre } \\
1,00_{\mathrm{a}}\end{array}$} & & \multirow{2}{*}{\begin{tabular}{|l} 
Nombre \\
$1,00_{\mathrm{a}}$
\end{tabular}} & & \multirow{2}{*}{$\begin{array}{r}\text { Nombre } \\
0,00\end{array}$} \\
\hline CSP & Agriculteur & & & & & & & & & & \\
\hline & Artisan-com-chef ent & & $1,00_{\mathrm{a}}$ & & $2,00_{\mathrm{a}}$ & & $1,00_{\mathrm{a}}$ & & 0,00 & & 0,00 \\
\hline & Cadre & & $18,00_{a}$ & & $23,00_{a, b}$ & & $11,00_{b}$ & & $29,00_{a}$ & & $13,00_{\mathrm{a}}$ \\
\hline & Prof intermédiaire & & $2,00_{\mathrm{a}}$ & & $4,00_{\mathrm{a}}$ & & $2,00_{\mathrm{a}}$ & & $4,00_{\mathrm{a}}$ & & $1,00_{\mathrm{a}}$ \\
\hline & Employé & & $8,00_{\mathrm{a}}$ & & $8,00_{a}$ & & $5,00_{a}$ & & $13,00_{\mathrm{a}}$ & & $3,00_{\mathrm{a}}$ \\
\hline & Ouvrier & & 0,00 & & $2,00_{\mathrm{a}}$ & & $1,00_{\mathrm{a}}$ & & $1,00_{\mathrm{a}}$ & & 0,00 \\
\hline & Etudiant & & $5,00_{\mathrm{a}}$ & & $30,00_{\mathrm{a}}$ & & $48,00_{b}$ & & $17,00_{\mathrm{a}}$ & & $9,00_{\mathrm{a}}$ \\
\hline & Retraité & & 0,00 & & $2,00_{\mathrm{a}}$ & & 0,00 & & $7,00_{\mathrm{a}}$ & & 0,00 \\
\hline & Sans emploi & & 0,00 & & $3,00_{\mathrm{a}}$ & & $1,00_{\mathrm{a}}$ & & $2,00_{\mathrm{a}}$ & & 0,00 \\
\hline \multirow[t]{2}{*}{ Sexe } & $\mathrm{H}$ & & $9,00_{\mathrm{a}}$ & & $21,00_{\mathrm{a}}$ & & $28,00_{\mathrm{a}}$ & & $21,00_{\mathrm{a}}$ & & $10,00_{\mathrm{a}}$ \\
\hline & $\mathrm{F}$ & & $26,00_{\mathrm{a}}$ & & $55,00_{\mathrm{a}}$ & & $43,00_{\mathrm{a}}$ & & $57,00_{\mathrm{a}}$ & & $19,00_{\mathrm{a}}$ \\
\hline \multicolumn{12}{|c|}{ Préférences de magasinage } \\
\hline \multirow{6}{*}{$\begin{array}{l}\text { Préférence pour } \\
\text { le plein }\end{array}$} & Drive & & $21,00 \mathrm{a}$ & & $1,00_{b}$ & & $1,00_{b}$ & & $2,00_{b}$ & & $13,00_{a}$ \\
\hline & Scannette & & 0,00 & & $3,00_{\mathrm{a}}$ & & $2,00_{\mathrm{a}}$ & & 0,00 & & $8,00_{\mathrm{b}}$ \\
\hline & Caisse express & & 0,00 & & $1,00_{\mathrm{a}}$ & & $6,00_{\mathrm{a}}$ & & $1,00_{\mathrm{a}}$ & & $1,00_{\mathrm{a}}$ \\
\hline & Caisse classique & & $9,00_{a}$ & & $63,00_{b}$ & & $55,00_{b}$ & & $61,00_{b}$ & & $7,00 \mathrm{a}$ \\
\hline & Magasin proximité & & 0,00 & & 0,00 & & $1,00_{\mathrm{a}}$ & & 0,00 & & 0,00 \\
\hline & Marché - vente directe & & $5,00_{a}$ & & $6,00_{a}$ & & 0,00 & & $9,00_{\mathrm{a}}$ & & 0,00 \\
\hline \multirow{6}{*}{$\begin{array}{l}\text { Préférence pour } \\
\text { le dépannage }\end{array}$} & Drive & & 0,00 & & 0,00 & & $1,00_{\mathrm{a}}$ & & 0,00 & & $2,00_{\mathrm{a}}$ \\
\hline & Scannette & & 0,00 & & 0,00 & & 0,00 & & 0,00 & & $4,00_{\mathrm{a}}$ \\
\hline & Caisse express & & $7,00_{\mathrm{a}, \mathrm{d}, \mathrm{e}}$ & & $28,00_{a, b}$ & & $48,00_{c}$ & & $7,00_{d}$ & & $15,00_{\mathrm{b}, \mathrm{c}, \mathrm{e}}$ \\
\hline & Caisse classique & & $5,00_{\mathrm{a}, \mathrm{c}, \mathrm{d}}$ & & $19,00_{a, b}$ & & $3,00_{\mathrm{c}}$ & & $21,00_{b, d}$ & & $4,00_{\mathrm{a}, \mathrm{c}, \mathrm{d}}$ \\
\hline & Magasin proximité & & 0,00 & & $2,00_{\mathrm{a}}$ & & 0,00 & & 0,00 & & 0,00 \\
\hline & Marché - vente directe & & $2,00_{\mathrm{a}}$ & & $4,00_{\mathrm{a}}$ & & 0,00 & & $2,00_{\mathrm{a}}$ & & 0,00 \\
\hline \multirow{6}{*}{$\begin{array}{l}\text { Préférence en cas } \\
\text { de produits } \\
\text { encombrants ou } \\
\text { lourds }\end{array}$} & Drive & & $19,00_{a}$ & & $5,00_{b}$ & & $4,00_{\mathrm{b}}$ & & $5,00_{b}$ & & $17,00_{a}$ \\
\hline & Scannette & & 0,00 & & $4,00_{\mathrm{a}}$ & & $2,00_{\mathrm{a}}$ & & 0,00 & & $6,00_{\mathrm{b}}$ \\
\hline & Caisse express & & 0,00 & & $3,00_{a}$ & & $9,00_{\mathrm{a}}$ & & 0,00 & & $1,00_{\mathrm{a}}$ \\
\hline & Caisse classique & & $12,00_{\mathrm{a}}$ & & $59,00_{b}$ & & $49,00_{b}$ & & $59,00_{b}$ & & $5,00_{\mathrm{a}}$ \\
\hline & Magasin proximité & & $2,00_{\mathrm{a}}$ & & $1,00_{\mathrm{a}}$ & & $2,00_{\mathrm{a}}$ & & $2,00_{\mathrm{a}}$ & & 0,00 \\
\hline & Marché - vente directe & & $2,00_{\mathrm{a}}$ & & $1,00_{\mathrm{a}}$ & & 0,00 & & $2,00_{\mathrm{a}}$ & & 0,00 \\
\hline
\end{tabular}

Tableau 2 -Typologie d'acheteurs alimentaires en 5 profils 


\section{Discussion : apports, limites et voies de recherche}

Cette dernière section synthétise les résultats, puis suggère des implications managériales pour les distributeurs. Enfin, à partir des limites de cette recherche, des pistes pour de futurs travaux académiques sont envisagées.

\subsection{Synthèse des résultats et apports de la recherche}

Sur le plan conceptuel, cette recherche plaide pour une meilleure prise en compte de la perception du temps présent dans les travaux en distribution. L'antagonisme «pressé/pas pressé » ne suffit pas pour analyser les modes de magasinage dans le contexte actuel, imprégné des nouvelles technologies. Il faut également tenir compte de l'acceptation des technologies et également du choix d'être pressé ou non. Cette recherche a permis d'aller audelà de cet antagonisme binaire en mettant en exergue cinq profils d'individus avec des rapports au temps plus complexes (tableau 3). Cette typologie permet de prendre en compte une réalité nuancée du temps subjectif en la reliant avec l'attitude à l'égard de la technologie.

\begin{tabular}{|c|c|c|c|c|c|}
\hline Profil & $\begin{array}{l}\text { Pression } \\
\text { temporelle } \\
\text { chronique }\end{array}$ & $\begin{array}{c}\text { Attitude / } \\
\text { technologie }\end{array}$ & $\begin{array}{c}\text { Caractéristiques } \\
\text { socio } \\
\text { démographiques }\end{array}$ & $\begin{array}{c}\text { Modes de } \\
\text { magasinage } \\
\text { fréquents }\end{array}$ & $\begin{array}{c}\text { Préférence } \\
\text { par situation } \\
\text { d'achat }^{10}\end{array}$ \\
\hline \multirow[t]{3}{*}{$\begin{array}{l}1-\text { Familles } \\
\text { tiraillées }\end{array}$} & \multirow[t]{3}{*}{ Pressés } & \multirow{3}{*}{$\begin{array}{l}\text { N'aiment pas } \\
\text { la } \\
\text { technologie, } \\
\text { s'y sentent } \\
\text { dépendants }\end{array}$} & \multirow[t]{3}{*}{$\begin{array}{c}\text { CSP }+, 3 \\
\text { personnes et }+\mathrm{au} \\
\text { foyer }\end{array}$} & Drive & $\begin{array}{c}\text { Plein }+ \\
\text { produits } \\
\text { encombrants }\end{array}$ \\
\hline & & & & Marchés & \\
\hline & & & & $\begin{array}{l}\text { Magasins de } \\
\text { proximité }\end{array}$ & \\
\hline \multirow[t]{2}{*}{$\begin{array}{l}2-\quad \text { Speed } \\
\text { technophobes }\end{array}$} & \multirow[t]{2}{*}{$\begin{array}{c}\text { Pressés et } \\
\text { fiers de l'être }\end{array}$} & \multirow{2}{*}{$\begin{array}{c}\text { N'aiment pas } \\
\text { la } \\
\text { technologie, } \\
\text { s'y sentent } \\
\text { dépendants }\end{array}$} & \multirow[t]{2}{*}{$\begin{array}{l}\text { Pas de } \\
\text { caractéristique } \\
\text { prédominante }\end{array}$} & $\begin{array}{l}\text { Caisses } \\
\text { classiques }\end{array}$ & $\begin{array}{c}\text { Plein }+ \\
\text { produits } \\
\text { encombrants }\end{array}$ \\
\hline & & & & Marchés & \\
\hline \multirow{2}{*}{$\begin{array}{l}3 \quad-\quad \text { Jeunes } \\
\text { adeptes du } \\
\text { dépannage }\end{array}$} & \multirow{2}{*}{$\begin{array}{c}\text { Non pressés, } \\
\text { détestent être } \\
\text { pressés }\end{array}$} & \multirow{2}{*}{$\begin{array}{c}\text { Aiment la } \\
\text { technologie, } \\
\text { fortement } \\
\text { accro }\end{array}$} & \multirow{2}{*}{$\begin{array}{l}\text { Jeunes adultes, } \\
\text { étudiants, } \\
\text { célibataires }\end{array}$} & $\begin{array}{l}\text { Caisses } \\
\text { express }\end{array}$ & Dépannage \\
\hline & & & & $\begin{array}{l}\text { Magasins de } \\
\text { proximité }\end{array}$ & \\
\hline \multirow[t]{3}{*}{$\begin{array}{l}4 \underset{1}{4} \quad \text { Zens } \\
\text { traditionnels }\end{array}$} & \multirow{3}{*}{$\begin{array}{c}\text { Non pressés, } \\
\text { détestent être } \\
\text { pressés }\end{array}$} & \multirow{3}{*}{$\begin{array}{c}\text { Ni goût, ni } \\
\text { dépendance à } \\
\text { la } \\
\text { technologie }\end{array}$} & \multirow[t]{3}{*}{$\begin{array}{l}\text { CSP+, les plus } \\
\text { âgés }\end{array}$} & $\begin{array}{l}\text { Magasins de } \\
\text { proximité }\end{array}$ & \\
\hline & & & & $\begin{array}{c}\text { Caisses } \\
\text { classiques }\end{array}$ & $\begin{array}{c}\text { Plein }+ \\
\text { produits } \\
\text { encombrants+ } \\
\text { dépannage }\end{array}$ \\
\hline & & & & Marchés & \\
\hline \multirow[t]{3}{*}{5 - Geeks } & \multirow{3}{*}{$\begin{array}{c}\text { Non pressés, } \\
\text { mais aiment } \\
\text { quand ils le } \\
\text { sont }\end{array}$} & \multirow{3}{*}{$\begin{array}{c}\text { Aiment la } \\
\text { technologie } \\
\text { sans se sentir } \\
\text { dépendants } \\
\text { d'elle }\end{array}$} & \multirow{3}{*}{$\begin{array}{c}\text { Pas de } \\
\text { caractéristique } \\
\text { prédominante }\end{array}$} & Scannette & \\
\hline & & & & $\begin{array}{l}\text { Caisses } \\
\text { express }\end{array}$ & Dépannage \\
\hline & & & & Drive & $\begin{array}{c}\text { Plein + } \\
\text { produits } \\
\text { encombrants }\end{array}$ \\
\hline
\end{tabular}

Tableau 3 - Synthèse des caractéristiques des 5 profils d'acheteurs alimentaires.

\footnotetext{
${ }^{10}$ Cette colonne recense pour chacun des modes de magasinage les plus fréquents, les situations d'achat lors desquelles ils sont préférés par les consommateurs du segment. Les cases grises signifient un mode de magasinage fréquent mais n'ayant pas la préférence des consommateurs dans une situation particulière.
} 


\subsection{Implications managériales pour la communication des distributeurs alimentaires}

Les résultats suggèrent des implications managériales pour les distributeurs. Ce travail suggère d'adapter le ciblage des services proposés. Ainsi, les caisses express sont utilisées par deux segments de clientèle aimant la technologie. Le côté ludique de ces caisses semble ainsi intéressant à mettre en avant, d'autant plus que des recherches antérieures ont montré qu'une perte de temps peut être ressentie par les utilisateurs (Mencarelli et Rivière, 2014). Etant donné que les «geeks » et les «jeunes adeptes du dépannage » ne sont pas des individus pressés dans leur quotidien, le côté ludique de scanner soi-même ses produits à la caisse semble être l'argument à développer. Ces arguments relatifs au fait de «jouer en faisant ses courses » peuvent être réitérés pour la scannette, qui séduit également le profil des «geeks ». Les arguments de communication relatifs au drive peuvent être adaptés à la cible que le distributeur souhaite privilégier. En effet, le drive apparait comme une formule de magasinage adoptée par deux segments opposés : les «familles tiraillées » (très pressées; n'aimant pas la technologie mais s'y sentant dépendantes) et les «geeks » (non pressés, aimant la technologie mais ne ressentant pas de dépendance vis-à-vis d'elle). L'argument de la praticité peut convenir néanmoins aux deux cibles, qui affirment préférer le drive dans des situations particulières d'achat, notamment en cas de produits encombrants ou lourds. Cet argument de communication pourra être complété par le côté amusant, ludique lié à la technologie du drive pour un distributeur souhaitant cibler davantage les «geeks». Pour toucher les «familles tiraillées », le gain de temps engendré par le drive est à mettre en avant. C'est ce qui est fait dans la plupart des cas aujourd'hui. Cette recherche propose ainsi des alternatives (praticité, côté ludique) pour les distributeurs souhaitant élargir la cible de leurs services de drive et se différencier de leurs concurrents.

Les magasins de proximité séduisent davantage de shoppers (trois profils sur cinq). Les «familles tiraillées» et les «zens traditionnels»y voient un moyen simple et efficace d'effectuer des courses alimentaires qui ne nécessite pas l'utilisation de toutes ces technologies qu'ils essaient de fuir dès que possible. Les "jeunes adeptes du dépannage », quant à eux y voient une manière de combler leur besoin récurrent de courses de dépannage par ce format spécifique bien que concurrencé par les caisses express. Enfin, les marchés et ventes directes permettent aux «speed technophobes», «zens traditionnels» et «familles tiraillées » de s'échapper du quotidien dicté par les technologies. Les courses sont alors un moyen de prendre son temps, de se focaliser sur la qualité du temps et non la quantité de temps passé à faire les courses. Ces arguments peuvent être mis en avant par les producteurs ou les communes pour promouvoir leurs marchés locaux.

Ainsi, en lieu et place d'un argument unique centré sur le gain de temps, indépendamment du format ou service à promouvoir (scannette, caisse express, drive, format de proximité), cette recherche propose des arguments alternatifs en fonction du profil ciblé (tableau 4).

\begin{tabular}{|l|l|l|}
\hline Mode de magasinage & \multicolumn{1}{|c|}{ Argument de communication } & \multicolumn{1}{c|}{ Cible visée } \\
\hline Scannette & Côté ludique & Geeks \\
\hline Caisse express & $\begin{array}{l}\text { Côté ludique - situation d'achat de } \\
\text { dépannage }\end{array}$ & Geeks, jeunes adeptes du dépannage \\
\hline \multirow{2}{*}{ Drive } & $\begin{array}{l}\text { Praticité - facilité pour le plein, les } \\
\text { produits encombrants }\end{array}$ & Geeks, Familles tiraillées \\
\cline { 2 - 3 } & Côté ludique & Geeks \\
\cline { 2 - 3 } & Gain de temps & Familles tiraillées \\
\hline Magasin de proximité & Simplicité (pas de technologie) & Familles tiraillées, Zens traditionnels \\
\cline { 2 - 3 } & $\begin{array}{l}\text { Efficacité, rapidité, choix restreint } \\
\text { (facilité de choix) }\end{array}$ & $\begin{array}{l}\text { Jeunes adeptes du dépannage, } \\
\text { Familles tiraillées, Zens traditionnels }\end{array}$ \\
\hline
\end{tabular}




\begin{tabular}{|l|l|l|}
\hline Marché, vente directe & $\begin{array}{l}\text { Retour aux sources, } \\
\text { Prendre son temps }\end{array}$ & $\begin{array}{l}\text { Speed technophobes, Zens } \\
\text { traditionnels, Familles tiraillées }\end{array}$ \\
\hline
\end{tabular}

Tableau 4 - Promouvoir un mode de magasinage alimentaire, des alternatives au « gain de temps »

\subsection{Limites et perspectives de recherche futures}

Malgré la volonté de mieux tenir compte des variables liées au «temps subjectif » dans le comportement de magasinage, cette recherche présente des limites. La principale tient à sa nature exploratoire et son échantillon restreint. De plus, le mode d'administration (en ligne) nécessitait une maîtrise des bases d'internet, ce qui constitue une limite à cette recherche. Néanmoins, la présence de diverses générations de manière équitable et le fait de s'intéresser au goût pour les technologies (non à leur maîtrise) permettent de contrebalancer cette limite.

Pour des recherches futures, il serait intéressant de répliquer cette étude dans d'autres pays ayant une culture temporelle différente et des technologies plus ou moins ancrées dans le quotidien. A ce titre, Diallo et al (2015) constatent qu'au Sénégal, l'accès à internet peut être perçu comme un luxe. De ce fait, la gratuité du réseau Wifi dans un centre commercial constitue une innovation technologique importante et plébiscitée. Ainsi, l'attitude à l'égard de la technologie risque d'être sensiblement différente chez ces consommateurs. Il serait également pertinent de lier cette typologie à d'autres variables temporelles structurelles, notamment l'orientation temporelle dans une zone précise (passé, présent ou futur). Une vision temporelle du comportement des consommateurs ne peut être que bénéfique et assurer aux distributeurs une meilleure prise en compte des besoins et attentes des acheteurs.

Ainsi, les recherches futures dans un contexte culturel français pourront étudier plus en profondeur certains segments de cette typologie. Tout d'abord, les zens traditionnels, les plus âgés, relativement neutres vis-à-vis de la technologie et peu pressés dans leur quotidien peut être intéressant. En effet, n'étant pas réfractaires aux technologies, ils privilégient les modes de magasinage traditionnels mais semblent avoir du temps pour s'initier aux nouveaux services de la grande distribution. Ce constat est à mettre en parallèle avec la littérature qui souligne les différences générationnelles dans l'adoption du comportement omni-canal et la nécessité d'accompagnement de la part des enseignes pour assurer la montée en compétences des consommateurs (Collin-Lachaud et Longo, 2014). Des démonstrations et conseils par le personnel pour limiter les coûts d'apprentissage pourraient ainsi progressivement susciter l'intérêt pour les modes de magasinage plus technologiques. Ensuite, concernant les «jeunes adeptes du dépannage », il conviendrait d'étudier les motivations et les freins à l'utilisation des formats de proximité par ces consommateurs autonomes depuis peu dans leurs choix alimentaires. Souhaiteraient-ils davantage de technologie dans ces magasins ?

Dans une autre perspective, d'autres voies de recherche peuvent être envisagées concernant l'innovation dans la grande distribution. Les recherches futures pourraient effectivement se concentrer sur différentes technologies SST présentes dans une même formule de vente. En ce sens, le format de l'hypermarché, qui concentre généralement différentes technologies, peut être un terrain intéressant pour une recherche couplant l'étude de variables dispositionnelles stables à des variables situationnelles (usage des technologies en magasin). Aussi, cette recherche amène à une réflexion sur le développement de nouveaux services. Pour les «geeks », aimant la technologie et les nouveaux services, il serait intéressant d'étudier l'appétence pour les SST les plus récentes ou à venir, telles que le paiement via smartphone ou l'utilisation du smartphone à la place de la scannette. Ils semblent effectivement être les early adopters des innovations de la distribution. Il apparaît cependant difficile de les identifier à l'aide de critères socio-démographiques, comparativement à d'autres profils. Les distributeurs pourraient repérer ces geeks sur la base de données comportementales relatives à 
leurs modes de magasinage lorsqu'ils possèdent une carte de fidélité (utilisation quasiexclusive de modes impliquant des technologies, date de la première utilisation de ces services au regard de la date de l'inauguration de ces services en point de vente...). Repérer ces individus pourrait servir pour les faire tester de nouveaux services en avant première, voire pour les solliciter dans une optique de co-création des modes de magasinage de demain. Enfin, il serait intéressant d'approfondir l'adéquation possible de certains profils avec des services hybrides comme le drive fermier. Ce drive fermier pourrait-il, par exemple, réconcilier les familles tiraillées avec les courses alimentaires, en répondant à la fois à leur besoin de tradition et à leur côté pressé, dépendant de la technologie ? Qu'en est-il des « speed technophobes », ayant l'habitude des marchés, mais pas du drive ?

\section{Conclusion}

La typologie proposée dans cet article, fondée sur des fréquences d'utilisation de modes de magasinage et des traits de personnalité stables relatifs au rapport au temps et à la technologie permet de répondre aux interrogations soulevées au départ. Ainsi, il ne faut pas nécessairement être pressé pour adopter les innovations de la distribution. Même si les distributeurs argumentent sur le gain de temps lié aux nouvelles technologies, il apparait que :

- Plus vite ne signifie pas forcément plus « geek », c'est-à-dire que les innovations de la grande distribution dans les technologies liées à la vente (scannette, caisse express) ne sont pas choisies par les consommateurs pressés.

- Les caisses classiques des hypermarchés, les magasins de proximité et le drive sont les modes de distribution privilégiés des familles pressées. Le gain de temps semble alors un argument plus pertinent pour les modes d'accès au client (drive, magasin de proximité) que pour les technologies de vente (caisses express, scannette).

Aussi, les formules proposées par la grande distribution étant plus ou moins technologiques, l'attitude à l'égard de la technologie joue un rôle dans le choix d'un mode de magasinage alimentaire en complément du fait d'être pressé ou non. Ainsi, plus que la recherche de rapidité, c'est le goût pour les technologies qui est davantage à l'origine du choix des selfservices technologies par les consommateurs qui les adoptent.

\section{Bibliographie}

AUBERT N. (2003), Le culte de l'urgence : la société malade du temps, Flammarion, Paris.

BEATTY S. E. et FERRELL M. E. (1998), «Impulsive buying: modeling its precursors », Journal of Retailing, Vol.74, n², p.169-191.

BERGADAA M. (1988), «Le temps et le comportement de l'individu (Part 1)», Recherche et Applications en Marketing, Vol.3, n4, p.57-72.

BERGADAA M. (1989), «Le temps et le comportement de l'individu (Part 2)», Recherche et Applications en Marketing, Vol.4, ${ }^{\circ} 1$, p.37-55.

BERRY L. (1979), «The time-buying consumer », Journal of Retailing, Vol. 55, n²4, p.58-69.

BOSNJAK M., GALESIC M. et TUTEN T. (2007), «Personality determinants of online shopping: explaining online purchase intentions using a hierarchical approach », Journal of Business Research, Vol.60, nº, p.597-605.

CHETTHAMRONGCHAI P et DAVIES G. (2000), « Segmenting the market for food shoppers using attitudes to shopping and to time », British Food Journal, Vol.102, n², p.81-101.

COLLIN-LACHAUD I. et LONGO C. (2014), «La montée en compétences du consommateur cross-canal : quelles conséquences pour les distributeurs ? », In COLLIN-LACHAUD I. (Ed.), Repenser le commerce, vers une perspective socio-culturelle de la distribution, Editions EMS Management \& Société, Cormelles Le Royal, p.199-220. 
COUTELLE P. et DES GARETS V. (2013), «Innovation technologique et relation client : Analyse des résistances des consommateurs aux technologies self-service », Actes du $16^{\text {ème }}$ Colloque Etienne Thil, Paris, 2-4 Octobre.

DABHOLKAR P.A., BOBBITT M. et LEE E.J. (2003), «Understanding consumer motivation and behavior related to self-scanning in retailing: implications for strategy and research on technology based self-service », International Journal of Service Industry Management, Vol.14, n¹, p.59-95.

DAUCE B. et RIEUNIER S. (2002), «Le marketing sensoriel du point de vente », Recherche et Applications en Marketing, Vol.17, $\mathrm{n}^{\circ} 4, \mathrm{p} .45-65$.

DIALLO, M.F., SECK, A.M. et SALL F.D. (2015), «L'innovation perçue et ses conséquences dans les centres commerciaux modernes d'Afrique : l'exemple du Sénégal », Management \& Avenir, $\mathrm{n}^{\circ} 81, \mathrm{p} .57-79$.

FAUCONNIER F. (2014), «Le click\&collect passé au crible », http://www.journaldunet.com/ebusiness/commerce/etude-click-and-collect.shtml

GAHINET M-C. (2014), «Les nouveaux formats alimentaires de proximité : regards croisés des distributeurs et des consommateurs », Management \& Avenir, ${ }^{\circ} 71, \mathrm{p} .153-168$.

GALLOUJ C. (2006), «Innovations et changements institutionnels dans le grand commerce :

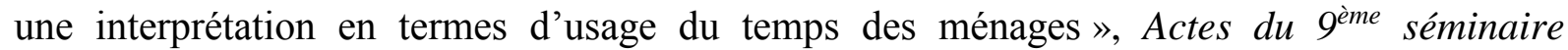
international de recherche en management des activités de service, Université Paul Cézanne, Aix-en-Provence.

GAUTSCHI D.A. (1981), «Specification of patronage models for retail center choice », Journal of Marketing Research, Vol.18, n², p.162-174.

GOUDEY A. (2013), «Exploration des effets du degré de technologie perçu du magasin sur le comportement de magasinage », Management \& Avenir, ${ }^{\circ} 63$, Vol.5, p.15-35.

GOURMELEN A. (2013), La pression temporelle ultime : conceptualisation et influence sur les motivations au bénévolat des retraités, Thèse de doctorat en Sciences de Gestion, Université de Bretagne Occidentale, Brest.

GOURMELEN A., URIEN B. et LE GALL-ELY M. (2016), «Le concept de pression temporelle ultime et sa mesure », Recherche et applications en marketing, Vol.31, $\mathrm{n}^{\circ} 4$, first published online, doi: 10.1177/0767370116635087.

HAIR J.F., BLACK W.C., BABIN B.J. et ANDERSON R.E. (2010), «Grouping data with cluster analysis », In Multivariate Data Analysis, $7^{\text {ème }}$ edition, Prentice Hall, p.505-564.

HERRINGTON J.D. et CAPELLA L.M. (1995), «Shopper reactions to perceived time pressure », International Journal of Retail \& Distribution Management, Vol.23, n ${ }^{\circ} 12$, p.13-21. IYER E. (1989), «Unplanned purchasing: knowledge of shopping environment and time pressure », Journal of Retailing, Vol.65, $\mathrm{n}^{\circ} 1, \mathrm{p} .40-58$.

JACOBY J., CHESNUT R.W., WEIGL K. et FISHER W.A. (1976), «Prepurchase information acquisition: description of a process methodology, research paradigm, and pilot investigation », Advances in Consumer Research, $\mathrm{n}^{\circ} 3, \mathrm{p} .306-314$

KAUFMAN-SCARBOROUGH C. et LINDQUIST J. D. (2003), «Understanding the experience of time scarcity », Time \& Society, Vol.12, n²-3, p.349-370.

KIM H.Y. et KIM Y.K. (2008), « Shopping enjoyment and store shopping modes: The moderating influence of chronic time pressure », Journal of Retailing and Consumer Services, Vol.15, p.410-419.

LALLEMENT J. (2010), «L'impact de la pression temporelle sur le traitement des informations », Recherche et Applications en Marketing, Vol.25, n4, p.45-68.

LALLEMENT J. et MACHAT S. (2011), «Au secours, tout va trop vite ! Nécessité d'une conceptualisation marketing de la pression temporelle chronique », Actes du $27^{\text {ème }}$ congrès de l'Association Française du Marketing (AFM), Bruxelles. 
LALLEMENT J et ZOLLINGER M. (2013), «Vite et à tout prix ? ou l'importance du prix pour le consommateur pressé ». Décisions Marketing, n69, p.11-24.

LECCARDI C. (2011), «Accélération du temps, crise du futur, crise de la politique », Temporalités, ${ }^{\circ} 13, \mathrm{p} .1-13$.

LEE H. et WHITLEY E.A. (2002), « Time and information technology: temporal impacts on individuals, organisations, and society », The information Society, Vol.18, n ${ }^{\circ}$, p.235-240.

LIN J.S.C. et HSIEH P. (2006), « The role of technology readiness in customers' perception and adoption of self-service technologies », International Journal of Service Industry Management, Vol.17, n5, p.497-517.

MCDONALD W. (1994), «Time use in shopping: The role of personal characteristics », Journal of Retailing, Vol.70, n 4, p.345-366.

MENCARELLI R. et RIVIERE A. (2014), «La participation du client dans un contexte de self-service technologies, une approche par la valeur perçue », Revue Française de Gestion, n'241, p.13-30.

MEUTER M.L., OSTROM A. L, RONDTREE R.I. et BITNER M.J. (2000), «Self-service technologies: understanding customer satisfaction with technology-based service encounters », Journal of Marketing, Vol.64, n³, p.1-14.

PARK C.W., IYER E.S. et SMITH D.C. (1989), «The effects of situational factors on in-store grocery shopping behavior: the role of store environment and time available for shopping », Journal of Consumer Research, Vol.15, n 4, p.422-433.

PICOT-COUPEY K., CLIQUET G. et PETR C. (2010), «Hypermarché, cybermarché et courses alimentaires: quels projets d'usage par les consommateurs? » Actes de la $9^{\text {ème }}$ conférence internationale marketing trends, 22-24 janvier, Venise.

RASTEGARY H. et LANDY F.J. (1993), «The interactions among time urgency, uncertainty, and time pressure », In O. SVENSON et A. J. MAULE (Ed.), Time pressure and stress in human judgment and decision making, New York Plenum Press, p.217-235.

RENKO S. et DRUZIJANIC M. (2014). «Perceived usefulness of innovative technology in retailing: consumers' and retailers' point of view ». Journal of Retailing and Consumer Services, Vol.21, n5, p.836-843.

REYNOLDS K.E., GANESH J. et LUCKETT M. (2002), «Traditional malls vs. factory outlets: comparing shopper typologies and implications for retail strategy », Journal of Business Research, Vol. 55, n9, p. 687-696.

RIZKALLA A. N. (1989), "Sense of time urgency and consumer well-being: testing alternative causal models », Advances in consumer research, Vol.16, p.180-188.

ROSA H. (2010), Accélération, une critique sociale du temps, Editions la découverte, Paris.

ROSEN L.D., WHALING K., CARRIER L.M., CHEEVER N.A. et ROKKUM J. (2013), «The media and technology usage and attitudes scale: an empirical investigation », Computers in Human Behavior, Vol.29, nº, p.2501-2511.

RUIZ J.P., CHEBAT J.C. et HANSEN P. (2004), « Another trip to the mall: a segmentation study of customers based on their activities », Journal of Retailing and Consumer Services, Vol.11, n6, p.333-350.

SZOLLOS A. (2009), « Toward a psychology of chronic time pressure », Time \& Society, Vol.18, n²-3, p.332-350.

WEIJTERS B, RANGARAJAN D., FALK T. et SCHILLEWAERT N. (2007), « Determinants and outcomes of customers' use of self-service technology in a retail setting », Journal of Service Research, Vol.10, n 1, p.3-21.

WRIGHT P. (1974), " The harassed decision-maker-time pressures, distractions, and the use of evidence », Journal of Applied Psychology, Vol. 59, n5, p.555-561. 